\title{
Metastable Bose-Einstein Condensate in a Linear Potential
}

\author{
D. S. Naik, S. R. Muniz and C. Raman* \\ School of Physics, Georgia Institute of Technology, Atlanta, Georgia 30332
}

(Dated: November 13, 2018)

\begin{abstract}
We have created a Bose-Einstein condensate whose spin orientation is metastable. Condensates were transferred into a quadrupole magnetic trap, where Majorana transitions limited the lifetime to a few hundred milliseconds, about 30 times the trapping period. Atoms held in the trap frequently displayed a ring-shaped time-of-flight distribution. We speculate that such a ring could be either a quantized vortex or a feature of the Majorana loss dynamics in the quantum regime.

PACS numbers: 03.75.Mn, 03.75.Lm, 03.75.Nt, 32.80.Lg, 32.80.Pj
\end{abstract}

Matter has many states which are fascinating, but whose existence is only transient. Classic examples of metastability in macroscopic quantum systems are the "persistent" currents of superfluids and superconductors 1]. Quantum gases have been shown to exhibit a rich variety of metastable states, including spatial spin domains in Bose-Einstein condensates (BECs) 2], lattices of quantized vortices [3], as well as the molecular states populated in the vicinity of a Feshbach resonance [4]. In all these examples, the lifetime depends critically on atom-atom interactions. In this work, we report on the experimental observation of a trapped BEC whose spin orientation is metastable in an external, inhomogeneous magnetic field. The decay of the trapped condensate thus depends primarily on single atom physics. The trapping field can in principle be used to coherently control the coupling between spin and spatial wavefunctions.

We produced the metastable BEC by transferring atoms into a magnetic quadrupole trap (see Figure 1). This is the first realization of a three-dimensional linear potential for BECs. Due to the presence of the magnetic field zero at the center, atoms spontaneously flipped their spin and entered an untrapped state. However, this process was relatively slow, and our condensate could remain trapped for hundreds of milliseconds, about 30 times the characteristic oscillation time in the linear trap. Moreover, we frequently observed that atoms released from the trap had a ring-shaped time-of-flight distribution reminiscent of the expansion of vortex states. We speculate on possible mechanisms for the formation of this ring.

An atom moving through an inhomogeneous $B$-field can flip its spin through a nonadiabatic process. Such Majorana transitions are most significant when the magnitude of $\vec{B}$ is close to zero, as it is near a region of radius $b \sim 1 \mu \mathrm{m}$ dimension near the center of a quadrupole trap. If the de Broglie wavelength $\lambda \leq b$, as it is usually for thermal atoms, one may use a semiclassical picture where the atomic motion can be treated classically and the internal spin states are treated quantum-mechanically. For a BEC, however, one must treat the entire problem quantum mechanically since all atoms occupy a single wavefunction that extends over a region that is typically much larger than $b$. For atoms whose internal spin is $F$ and a three-dimensional quadrupole magnetic field $\vec{B}=B^{\prime}(x \hat{x}+y \hat{y}-2 z \hat{z})$, the resulting Hamiltonian contains the potential energy term:

$$
V=-\vec{\mu} \cdot \vec{B}(\vec{r})=\mu_{B} g_{F} B^{\prime}\left(x \hat{F}_{x}+y \hat{F}_{y}-2 z \hat{F}_{z}\right)
$$

where $\vec{F}=\left(\hat{F}_{x}, \hat{F}_{y}, \hat{F}_{z}\right)$ is the vector spin- $F$ operator and $\mu_{B}$ is the Bohr magneton. Since $V$ acts on both spin and spatial coordinates, the trapped and untrapped states are coupled to one another. In general, this process is completely coherent, and could be used as a mechanism for creating spinor condensates, as has been discussed for other inhomogeneous field geometries $[5]$. We note that the same coupling exists in Ioffe-Pritchard traps, but is much smaller due to the finite bias field $B_{z}$ that preserves the spin orientation.
A)

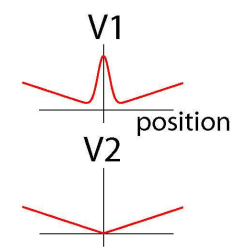

D)

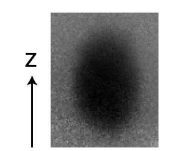

B)
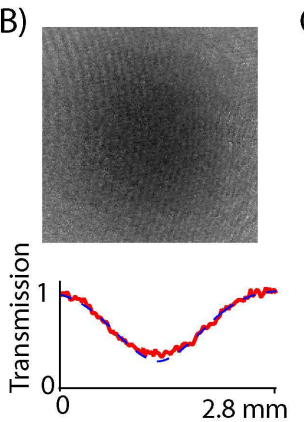

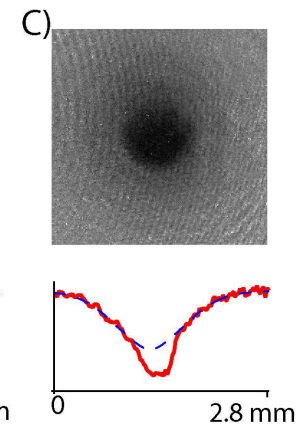

FIG. 1: (Color online). Metastable Bose-Einstein condensate. (A) Atoms are transferred from an optically plugged trap (potential V1) into a purely linear trap (potential V2) by slowly reducing the plug intensity to zero. (B) Transfer of purely thermal atoms cooled to a final rf value of $0.35 \mathrm{MHz}$, and (C) transfer of Bose-Einstein condensates at $0.25 \mathrm{MHz}$ rf. Each image was taken along the $\hat{z}$ axis after $20 \mathrm{~ms}$ TOF, and is 1.6 $\times 1.6 \mathrm{~mm}$. Below each image is a slice through the data as well as a fit to the thermal wings (dashed lines). (D) View perpendicular to the $\hat{z}$ axis shows anisotropic expansion of the BEC after $15 \mathrm{~ms}$ TOF. Image is $0.5 \times 0.7 \mathrm{~mm}$.

We begin by creating Bose-Einstein condensates of about $5 \times 10^{6}$ sodium atoms in the $F=1, M_{F}=-1$ state in an optically plugged quadrupole trap (OPT) as described in [6]. Majorana losses are suppressed by focusing a $40 \mu \mathrm{m}$, far-off resonance, blue-detuned, $532 \mathrm{~nm}$ "plug" laser beam onto the spatial location of the mag- 
netic field zero. After making a BEC, we ramp the intensity of the plug laser from an AC Stark shift of $60 \mu \mathrm{K}$ to zero over $200 \mathrm{~ms}$, leaving the condensate trapped by a pure quadrupole magnetic field whose axial field gradient could be varied. After a variable hold time, we shut off the quadrupole coils allowing for times-of-flight up to 35 milliseconds. Five to ten milliseconds before imaging, we turned on a $\hat{z}$-directed bias field of approximately 1 Gauss. Absorption imaging was performed on either the $F=1$ to $F^{\prime}=2$ transition using a $150 \mu$ s probe pulse or by first optically pumping the atoms into the $F=2$ ground state with a 1.5 to $4 \mathrm{~ms}$ pump pulse followed by a $150 \mu$ s probe pulse on the $F=2$ to $F^{\prime}=3$ cycling transition.

Figure 1 encapsulates our main result: we have produced a condensate in the purely linear potential of the quadrupole coils. That it merely exists is rather counterintuitive, since one might expect the atoms to rapidly leave the trap by Majorana transitions. In fact, as we show below, these transitions occur slowly when compared with the natural timescales of the system. We observed bimodal time-of-flight distributions (Figure 1c) when atoms were imaged along the symmetry axis of the quadrupole field $\hat{z}$ (which coincides with the direction of gravity). Due to the presence of the condensate, the fit to the thermal wings does not agree with the data in the center of the cloud. The fit agreed well, however, when we transferred a purely thermal cloud, as in Figure 1b. The BEC expanded anisotropically when viewed from the side (Figure 1d) due to the increased mean-field pressure arising from the factor of 2 larger axial magnetic field gradient compared with the radial $(\hat{x}$ or $\hat{y}$ ) direction.

Unlike a condensate in the Thomas-Fermi regime in a harmonic potential, the time-of-flight expansion does not obey a simple scaling of the parabolic distribution. In some cases, the entire shape of the distribution had changed dramatically, as we will discuss later. Therefore, we used a model-independent approach to analyze the images and extract both condensate number and thermal number. We removed the central region that contained mostly the condensate and fit a 2-dimensional thermal distribution to the remaining image. We deduced the condensate number by subtracting the total thermal number obtained from the fit from the total atom number obtained by summing the column density over the entire original image. The total number was adjusted for shot-to-shot background level fluctuations using the background obtained from the thermal fit.

We observed our condensate to have a lifetime of a few hundred milliseconds in the purely linear potential. We attribute the decay to Majorana transitions to an untrapped state, which should occur in a narrow region near the cloud center. By comparison, in an rf-shielded OPT, without Majorana loss, condensates were observed for more than 12 seconds. Figure 2 a shows the decay curve for traps with two different axial magnetic field
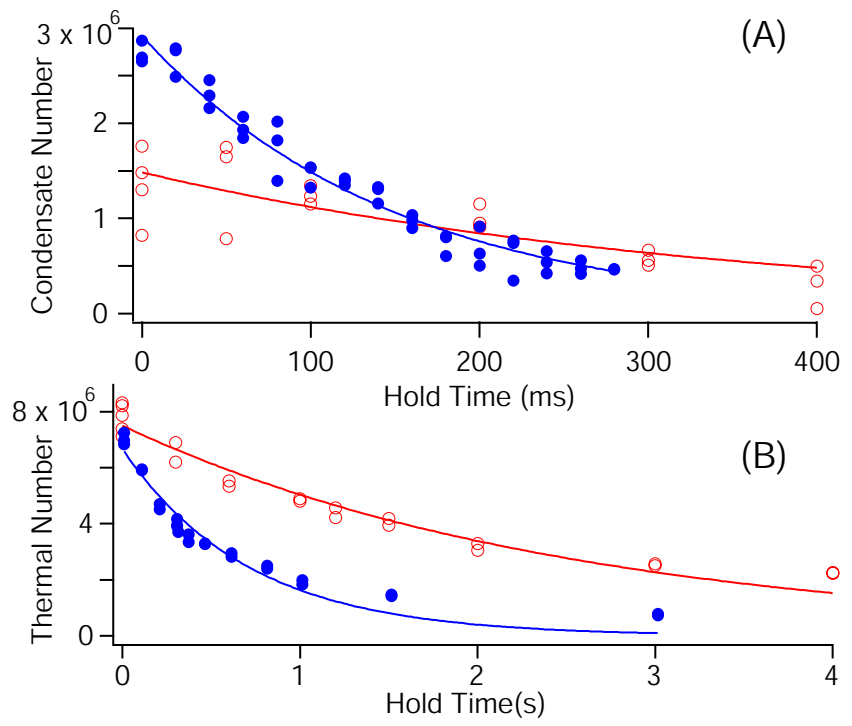

FIG. 2: (Color online). Decay in the linear trap. (A) Condensate number is plotted against hold time for traps with axial magnetic field gradients of $21 \mathrm{G} / \mathrm{cm}$ (filled circles) and 13 $\mathrm{G} / \mathrm{cm}$ (open circles). (B) Decay of purely thermal clouds with field gradients of $21 \mathrm{G} / \mathrm{cm}$ (open circles) and $105 \mathrm{G} / \mathrm{cm}$ (filled circles), corresponding to measured temperatures of $2.7 \mu \mathrm{K}$ and $8.2 \mu \mathrm{K}$, respectively. The solid lines are fits to a single exponential, as described in the text.

gradients, $21 \mathrm{G} / \mathrm{cm}$ and $13 \mathrm{G} / \mathrm{cm}$, along with a fit to a single exponential decay for each. The condensate fractions were $45 \%$ and $30 \%$, respectively, at zero hold time. The measured decay rates were $7 \mathrm{~s}^{-1}$ and $3 \mathrm{~s}^{-1}$, respectively. We can compare this rate to a WKB estimate of the oscillation frequency which in general depends on the size of the condensate [7]. The result is $\nu_{o s c}=\frac{1}{4 \sqrt{2}} \sqrt{\frac{\tilde{\mu} B^{\prime}}{M R}}$, where $\tilde{\mu}=\frac{1}{2} \mu_{B}$ is the magnetic moment and $B^{\prime}=\frac{2}{3} B_{z}^{\prime}$ is the spatially averaged field gradient. $R$ is the average condensate radius, which we estimate in the section below to be $\frac{3}{4} R_{\rho}=13.5 \mu \mathrm{m}$ for our $21 \mathrm{G} / \mathrm{cm}$ trap, where $R_{\rho}$ is the Thomas-Fermi radius in the $x-y$ plane. This yields $\nu_{\text {osc }}=200 \mathrm{~Hz}$, about 30 times faster than the rate of decay. Such a long lifetime was only possible by using such weak field gradients. In the case of the weaker trap with $13 \mathrm{G} / \mathrm{cm}$ gradient, the loss rate was smaller due to the larger condensate size, as discussed below. Indeed, we could observe a BEC for up to $0.5 \mathrm{~s}$ in this trap. Longer lifetimes could not be obtained by weakening the trap much further due to the difficulty of balancing gravity, which requires at least $8 \mathrm{G} / \mathrm{cm}$.

One may use a semiclassical model to estimate the Majorana rate, as in reference [8]. In this model, the rate of loss $\Gamma_{M}$ only depends on the cloud size,

$$
\Gamma_{M} \sim \frac{\hbar}{M R^{2}}
$$

and is simply related to the statistical probability that an atom trapped in a cloud of radius $R$ will pass directly through a hole of radius $b=\sqrt{\frac{\hbar v}{\tilde{\mu} B^{\prime}}}$ located at the origin. 
For a gas above the transition temperature, the scaling relation (2) was verified in previous work [8]. To compare with our system, we measured the loss rate for thermal clouds just above the transition temperature. The data are shown in Figure 2b for field gradients of $21 \mathrm{G} / \mathrm{cm}$ and $105 \mathrm{G} / \mathrm{cm}$, corresponding to temperatures of $2.7 \mu \mathrm{K}$ and $8.2 \mu \mathrm{K}$ and average cloud sizes of $R=\frac{k_{B} T}{\tilde{\mu} B^{\prime}}=56 \mu \mathrm{m}$ and $35 \mu \mathrm{m}$, respectively. We measured the loss rate by fitting the data to an exponential decay, which should be accurate at least for short times before the loss causes the cloud to heat up significantly. The theoretical prediction from Eqn. (2) for a gas at the same temperatures are $0.9 \mathrm{~s}^{-1}$ and $2.2 \mathrm{~s}^{-1}$, while we measured 0.4 and 1.4 $\mathrm{s}^{-1}$, respectively. This gives us confidence that the semiclassical scaling of Eqn. (2) yields the observed loss rate within a factor of about 2 .

For a Bose-Einstein condensate, the spin-flip occurs through a coherent process governed by the GrossPitaevskii (GP) equation for the wavefunction $\Psi$, which is a $2 F+1$ component spinor defined with respect to the space-fixed $\hat{z}$-axis. Solving this equation is in general quite difficult due to the coupling between spin and space which was discussed earlier. However, we gain considerable insight by viewing the problem in a spatially varying spin basis defined with respect to the local magnetic field direction [9, 10]. We apply a local transformation $\Lambda=\Lambda(\vec{r})$ that diagonalizes the potential energy term $V$ in spin space. The resulting GP equation is

$$
\left[\Lambda\left(\frac{\hat{p}^{2}}{2 M}\right) \Lambda^{-1}\right] \Phi+\left[g n+V_{d}-\mu\right] \Phi=0
$$

where $\mu$ is the chemical potential, $\Phi=\Lambda \Psi$, and $V_{d}=$ $\Lambda V \Lambda^{-1}$ is diagonal in the local spin basis. Its eigenvectors are the weak-field seeking, strong-field seeking and field-insensitive states, with eigenvalues $+\tilde{\mu}|\vec{B}(\vec{r})|$, $-\tilde{\mu}|\vec{B}(\vec{r})|$ and 0 , respectively. The trapped state is the weak-field seeking state. The nonlinear interaction term is $g n$, where $n$ is the particle density, $g=4 \pi \hbar^{2} a / M$ and $a$ is the two-body scattering length. We have neglected the spin dependence of this term. This is a valid assumption in our case since we are only interested in the evolution of the trapped state, upon which the low density untrapped particles should have little effect.

The second term of Eqn. (3), $\left[g n+V_{d}-\mu\right]$, is diagonal in the local spin basis and therefore describes a set of adiabatic potentials determined by the eigenvalues of $V_{d}$. However, since the kinetic energy operator does not commute with $\Lambda(\vec{r})$, the first term couples the different spin channels together, resulting in Majorana transitions.

One must solve the problem using a coupled channel approach similar to that involved in the theory of Feshbach resonances 11]. While such a solution is beyond the scope of this paper, we can compare the loss rate for our BEC using the semi-classical Eqn. (2). We use the Thomas-Fermi density distribution for the linear potential, which is a cone-shaped function: $n(x, y, z)=$
$n_{0}\left(1-\frac{1}{R_{\rho}} \sqrt{x^{2}+y^{2}+4 z^{2}}\right)$, where $n_{0}$ is the peak density. We can determine $n_{0}$ self-consistently using the relations $g n_{0}=\mu$ and $\mu=\tilde{\mu} B_{z}^{\prime} R_{\rho} / 2$ where $\mu$ is the chemical potential. For our $21 \mathrm{G} / \mathrm{cm}$ trap, $\mu \simeq 0.65 \mu \mathrm{K}$ yielding $R_{\rho}=18 \mu \mathrm{m}$ for $N=3 \times 10^{6}$ atoms. Taking the average radius to be $R=\frac{3}{4} R_{\rho}$ as we have earlier for the thermal cloud, we estimate that the condensate should decay at a rate which is $(56 / 13.5)^{2}=17$ times faster than the thermal cloud in the same trap. Using the experimental value of $0.4 \mathrm{~s}^{-1}$ from Figure $2 \mathrm{~b}$, we get $\Gamma_{M}=6.9 \mathrm{~s}^{-1}$, which compares fortuitously well with our measurement of 7 $\mathrm{s}^{-1}$. We conclude that the loss dynamics for both condensed and normal components are fairly similar, when the cloud sizes are scaled appropriately.

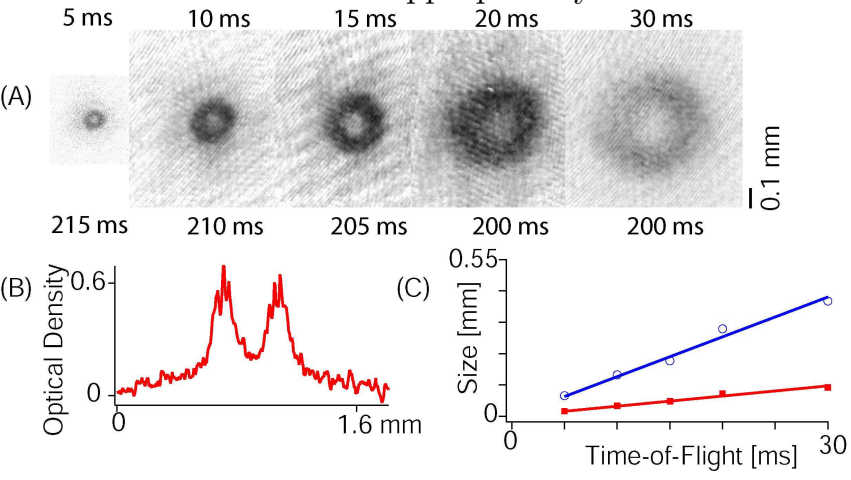

FIG. 3: (Color online). Ring shaped expansion of a BEC. (A) Images of a condensate after various hold times (given below each image) and times of flight (given above). (B) Slice through the data at $30 \mathrm{~ms}$ TOF shows a high-contrast density minimum on top of the thermal background. (C) Radius of the central hole (filled squares) and the condensate (open circles) versus time-of-flight. The velocities of expansion are $14 \mathrm{~mm} / \mathrm{sec}$ and $3.5 \mathrm{~mm} / \mathrm{sec}$, respectively.

While the loss rate is fairly well understood, the quantum dynamics of the trapped atoms are more complex. We have observed new, intriguing features of the condensate in the linear trap for which there is no current explanation, although a more detailed investigation is ongoing. After hold times of 100-200 ms, the time-of-flight distribution frequently displayed a dramatic and unexpected signature-a single, clear hole in the center of the cloud. This hole reflects a depletion of low momentum atoms in the image. Figure 3a shows a succession of images taken for different times-of-flight. We used $F=2$ imaging, which is spin-state independent. This established that the observed ring feature is a variation in the total atomic density and not due to the formation of possible spin domains that might have different optical absorption [12]. Accounting for the thermal background in the image, the density minimum in the center was typically $50 \%$ of the condensate peak density, but on some occasions could be about $90 \%$. The latter case is shown in Figure 3b. Ring-shaped condensates were visible in approximately half of the experimental runs, while for the remaining images the absorption profile was relatively smooth and showed little to no structure. Moreover, we 
could not easily see the ring in the images with no hold time. It was possibly obscured by high optical density. Similar results were obtained for traps of both $21 \mathrm{G} / \mathrm{cm}$ and $13 \mathrm{G} / \mathrm{cm}$, indicating it is a general feature of the linear potential.

We observed that the ring structure had appeared after only $5 \mathrm{~ms}$ of expansion. For each image in Figure 3a, we varied the detuning of the optical pumping light to control the number of atoms pumped into the $F=2$ state for imaging. This resulted in an optical depth close to 1 for each image without introducing spatial distortions. In Figure 3c, we have plotted the hole radius as well as the overall cloud radius as a function of the timeof-flight. To quantify these parameters, we fit the ring pattern from a slice such as shown in Figure $3 \mathrm{~b}$ to the sum of 2 Gaussians at locations $x_{1}, x_{2}$ with $1 / e$ half-widths $w_{1}, w_{2}$. The condensate radius was defined to be $R_{c}=$ $\frac{1}{2}\left(x_{2}-x_{1}+w_{1}+w_{2}\right)$, while the hole radius was $R_{h}=$ $\frac{1}{4}\left(x_{2}-x_{1}\right)$. Both $R_{h}$ and $R_{c}$ are plotted in Figure $3 \mathrm{c}$ and increase linearly with time, with $\frac{d R_{h}}{d t}$ a factor of 4 smaller.

Our observation of the ring pattern is unexpected, and we do not have a conclusive explanation for it as of yet. However, our data in Figure 3 clearly shows that the wavefunction of the condensed state before expansion has a complex structure. The ring feature we observe could be a vortex state-the core of a vortex should expand at a velocity which is roughly proportional to that of the overall condensate size 13], consistent with our observations. There could be two separate condensates formed in the optically plugged trap which are merged together when the plug is removed [6]. Phase fluctuations between the two uncorrelated BECs could create topological defects, including vortices. The random character of this process might explain why we do not observe the hole on every cycle of the experiment.

The stability of a vortex in a linear potential has not been explored theoretically. A rough estimate suggests, however, that the presence of a vortex should not interfere with the spin-flip process occurring near the trap center. This is due to the fact that the depletion of the superfluid density occurs only within a region of the size of the vortex core, typically $\approx 0.13 \mu \mathrm{m}$ for our system, considerably smaller than the size of the hole, $b=\sqrt{\frac{\hbar c}{\tilde{\mu} B^{\prime}}} \approx 1.5 \mu \mathrm{m}$, estimated from the semiclassical model [8] replacing the atomic velocity with that of sound propagation for our condensate, $c=1.5 \mathrm{~cm} / \mathrm{s}$. Indeed, we observed no correlation between observation of the hole and increased lifetime of the BEC.

One unique feature of the linear potential is that the magnetic field direction varies in space, unlike most IoffePritchard traps. Therefore, the condensate has an inhomogeneous spin orientation, and it is plausible that a vortex state could be created by the shut-off of the trap, when the inhomogeneous field is removed. We are investigating this mechanism further.
The spatially localized character of Majorana loss may also play a role in creating the pattern observed in Figure 3 Indeed, the time evolution is quite rich, as the initially stationary condensate begins to acquire an inward velocity field toward the magnetic field zero to balance the loss. Spatial patterns have been observed in electromagnetic wave propagation 14 and trapped BECs with negative scattering length 15], both examples of systems where nonlinearity effectively confines losses to a small region of space where the wave amplitude is highest. They bear a strong resemblance to our trapped BEC, where although the losses are linear in atom density, they are localized due to the magnetic field. Numerical simulations are necessary to shed more light on the dynamics of this novel state.

In summary, we have demonstrated metastable BoseEinstein condensates in a purely linear potential. Majorana losses are shown to have intriguing effects on the time-of-flight evolution. These could be signatures of vortex formation, which could be verified, for example, by interferometric means [16].

We thank Li You, T. A. B. Kennedy, P. di Trapani, M. S. Chapman and M. Bhattacharya for useful discussions. This work was supported by the DoE, ARO and by Georgia Tech.

* Electronic address: craman@gatech.edu

[1] D. R. Tilley and J. Tilley, Superfluidity and Superconductivity (Wiley, New York, 1990).

[2] H.-J. Miesner et al., Phys. Rev. Lett. 82, 2228 (1999).

[3] J. R. Abo-Shaeer, C. Raman, J. M. Vogels, and W. Ketterle, Science 292, 476 (2001).

[4] E. A. Donley et al., Nature 417, 529 (2002). M. Greiner et al., Nature 426,537 (2003). S. Jochim et al., Science 302, 2101 (2003). K. E. Strecker et al., Phys. Rev. Lett. 91, 080406 (2003). M. W. Zwierlein et al., Phys. Rev. Lett. 91, 250401 (2003).

[5] H. Pu et al., Phys. Rev. A 63, 063603 (2001).

[6] D. S. Naik and C. Raman, Phys. Rev. A 71, 033617 (2005).

[7] E. Merzbacher, Quantum Mechanics (John Wiley and Sons, Inc., New York, 1998).

[8] W. Petrich et al., Phys. Rev. Lett. 74, 3352 (1995).

[9] T. H. Bergeman et al., J. Opt. Soc. Am. B 6, 2249 (1989).

[10] H. J. Metcalf and P. v. d. Straten, Laser Cooling and Trapping (Springer, New York, 1999).

[11] H. Friedrich, Theoretical Atomic Physics (SpringerVerlag, Berlin, 1990).

[12] In any case, we do not see any evidence of untrapped atoms in the images due to their low density.

[13] E. Lundh et al., Phys. Rev. A 58, 4816 (1998).

[14] M. A. Porras et al., Phys. Rev. Lett. 93, 153902 (2004).

[15] M. Ueda et al., J. Phys. Soc. Jpn. 72, Suppl. C pp.127 (2003).

[16] S. Inouye et al., Phys. Rev. Lett. 87, 080402 (2001). 